\title{
Cytoprotective effects of myristicin against hypoxia-induced apoptosis and endoplasmic reticulum stress in rat dorsal root ganglion neurons
}

\author{
QUANLAI ZHAO, CHEN LIU, XIANG SHEN, LIANG XIAO, \\ HONG WANG, PING LIU, LINGTING WANG and HONGGUANG XU \\ Department of Orthopedic Surgery, Yijishan Hospital, Wannan Medical College, Wuhu, Anhui 241001, P.R. China
}

Received October 19, 2015; Accepted October 25, 2016

DOI: $10.3892 / \mathrm{mmr} .2017 .6258$

\begin{abstract}
The aim of the present study was to investigate the role of myristicin (Myr; 1-allyl-5-methoxy-3,4-methylenedioxybenzene), an active aromatic compound isolated from nutmeg, carrot, basil, cinnamon and parsley, in hypoxia-induced apoptosis in rat dorsal root ganglion (DRG) neurons. It was observed that Myr significantly enhanced cell viability in hypoxia-induced DRG neurons in a dose-dependent manner; the optimal concentration of Myr was $50 \mu \mathrm{M}$. Furthermore, Myr reduced the percentage of deoxynucleotidyl transferase-mediated dUTP nick end-labeling-positive neuronal cells and influenced the expression of the pro-apoptotic gene B-cell lymphoma 2 (Bcl-2) associated $\mathrm{X}$ protein, the apoptosis protease cleaved caspase- 3 and the anti-apoptotic gene Bcl-2, in the hypoxia-induced group. In addition, Myr protected against hypoxic injury in DRG neurons by inhibiting malondialdehyde and lactate dehydrogenase, however upregulating superoxide dismutase and glutathione peroxidase. Myr reduced the expression of endoplasmic reticulum stress (ERS) markers, including CCAAT/enhancer-binding protein-homologous protein, glucose-related protein 78 and cleaved caspase-12 in the hypoxia-induced group. To the best of our knowledge, this is the first demonstration of the activity of Myr against hypoxia-induced apoptosis in rat DRG neurons via inhibition of the ERS pathway.
\end{abstract}

\section{Introduction}

The causes of spinal cord injury, in response to hypoxic-ischemic insults, are multifactorial, and affect the central nervous system (CNS) $(1,2)$. In the developing CNS, lack of oxygen

Correspondence to: Dr Hongguang Xu, Department of Orthopedic Surgery, YijishanHospital,Wannan Medical College,24 Yinhu S Road, Wuhu, Anhui 241001, P.R. China

E-mail:xuhg@medmail.com.cn

Key words: Myristicin, hypoxia, dorsal root ganglion neurons, apoptosis, endoplasmic reticulum stress results in an initial depletion of high-energy phosphates, in particular adenosine triphosphate and phosphocreatine $(3,4)$. These levels transiently return to baseline, followed by a second, more prolonged depletion of cellular energy reserves accompanied by the progression of spinal cord and brain injury (2). Previous studies have revealed that mitochondria determine cell fate in neuronal cells (4-6). They may induce cell death via the release of pro-apoptotic proteins, which occurs following mitochondrial permeabilization (MP) (7). MP may occur through selective opening of the outer mitochondrial membrane, mitochondrial outer membrane permeabilization (MOMP), or through opening of the mitochondrial permeability transition pore, which permeabilizes the outer and inner mitochondrial membranes $(7,8)$. MOMP appears to predominantly induce apoptosis, whereas mitochondrial permeability transition pore opening results in mitochondrial swelling and may lead to necrotic cell death $(7,8)$.

Protein folding in the endoplasmic reticulum (ER) is impaired under various physical and pathological conditions, termed endoplasmic reticulum stress (ERS) (9). ERS, induced by the activation of the unfolded protein response (UPR), is characterized by the upregulation of molecular chaperone glucose-related protein 78 (GRP78) and the activation of apoptosis $(9,10)$. GRP78 associates with various critical transmembrane ER signaling proteins $(9,11)$. The UPR induces signals via three distinct stress sensors located at the ER membrane: Protein kinase RNA-like ER kinase (PERK), inositol-requiring protein-1 (IRE-1) and activating transcription factor-6 (ATF-6) (11). Of these, PERK, whose intrinsic kinase activity is induced by oligomerization, regulates the phosphorylation of the eukaryotic translation initiation factor $2 \alpha$, which induces the suppression of global mRNA translation to protect cells against ERS (12). Various ERS-associated signaling pathways have been proposed to be involved with this programmed cell death, including the activation of CCAAT/enhancer-binding protein homologous protein (CHOP) and caspase-12 (10,13). These studies suggested that the induction of ERS was closely associated with apoptosis. However, whether ERS is involved in hypoxia-induced apoptosis in rat dorsal root ganglion (DRG) neurons remains to be elucidated. 
Recent studies have focused on the activity of non-nutritional dietary compounds that have protective or disease preventive properties (14-16). Myristicin (Myr; 1-allyl-5-methoxy-3,4-methylenedioxybenzene) is an active aromatic compound present in nutmeg (the seed of Myristica fragrans), carrot, basil, cinnamon and parsley (15). Myr has been revealed to have antibacterial (16), hepatoprotective (17), anti-inflammatory (18) and anticancer (15) effects. Additionally, Myr has exhibited significant effects on the CNS. Myr may induce neurotoxicity in SK-N-SH human neuroblastoma cells (19). However, whether Myr induces this effect against hypoxia-induced apoptosis in rat DRG neurons remains to be elucidated.

The present study observed that Myr enhanced cell viability significantly in hypoxia-induced rat DRG neurons in a dose-dependent manner. In addition, Myr reduced the terminal deoxynucleotidyl transferase-mediated dUTP nick end-labeling (TUNEL)-positive DRG neurons and influenced the expression of apoptotic genes in the hypoxia-induced group. Furthermore, Myr exhibited a protective effect against hypoxic injury in DRG neurons, the underlying mechanism of which may be associated with the inhibition of the ERS pathway.

\section{Materials and methods}

Cell culture. Myr (CAS no. 60791 0) was obtained from Sigma-Aldrich; Merck Millipore (Darmstadt, Germany). The cell culture materials and reagents were obtained from Invitrogen; Thermo Fisher Scientific, Inc. (Waltham, MA, USA). DRG neuron culture was based on previous studies, including our own (20-22). Sprague-Dawley (SD) rats (n=24; weight, 25-30 g; male) were purchased from the Animal Laboratory of Wannan Medical College (Wuhu, China). Rats were kept on a $12 \mathrm{~h} / 12 \mathrm{~h}$ light-dark cycle and freely given food and water in a pathogen-free area. All procedures that involved animals were approved by the Institutional Animal Care and Use Committee of Yijishan Hospital (Wuhu, China). DRG neurons were harvested from rats at day 15 . The DRG neurons were digested with $0.25 \%$ trypsin in Hank's Balanced Salt solution (Invitrogen; Thermo Fisher Scientific, Inc.) at $37^{\circ} \mathrm{C}$ for $20 \mathrm{~min}$, and added to $10 \%$ fetal bovine serum to prevent digestion. Cells were passed through a 74- $\mu \mathrm{m}$ filter and centrifuged in $1,500 \mathrm{x} g$ at $4^{\circ} \mathrm{C}$ for $5 \mathrm{~min}$. The pellet was resuspended in a neurobasal medium of $2 \%$ B-27 supplement, $10 \mathrm{ng} / \mathrm{ml}$ nerve growth factor, $1 \mathrm{mmol} / 1 \mathrm{~L}$-glutamine and $1000 \mathrm{U} / \mathrm{ml}$ penicillin/streptomycin/neomycin solution. Dissociated DRG neurons were cultured in poly-D-lysine-precoated 6-well culture clusters at $2 \times 10^{6}$ cells/well in $2 \mathrm{ml}$. DRG neurons were cultured in media at $37^{\circ} \mathrm{C}$ with $5 \% \mathrm{CO}_{2}$ and maintained in media containing 20 $\mu \mathrm{mol} / \mathrm{l}$ floxuridine for another $24 \mathrm{~h}$ to inhibit the growth of non-neuronal cells. The purity of neuronal cells was confirmed by fluorescent labeling of neurofilament protein 200 (cat. no. orb18247; dilution, 1:600; Biorbyt Ltd., Cambridge, UK) and NeuN (cat no. 104225; dilution, 1:500, Abcam, Cambridge, UK) (data not shown). All experimental procedures were performed in accordance with the National Institute of Health Guidelines for the Care and Use of Laboratory Animals and were approved by the Animal Experimentation Committee of Wannan Medical College (Wuhu, China).
Analysis of cell viability. Cell viability was measured via a quantitative colorimetric assay with 3-(4,5-dimethylthiazol-2-yl)-2,5-diphenyltetrazolium bromide (MTT; Sigma-Aldrich; Merck Millipore). Briefly, DRG neurons were seeded into 96 -well plates at $5 \times 10^{4}$ cells per well. A portion of cells were treated with $1 \% \mathrm{O}_{2}$ (hypoxia) for $24 \mathrm{~h}$, and incubated with $5 \mathrm{mg} / \mathrm{ml}$ MTT solution for a further $4 \mathrm{~h}$. A total of $100 \mu \mathrm{l}$ dimethyl sulfoxide was added to each well and the absorbance was measured at a wavelength of $540 \mathrm{~nm}$ using a microplate reader (Bio-Rad Laboratories, Inc., Hercules, CA, USA). Cell viability was expressed as the ratio of clustered DRG neurons to control group The control group were cultured in neurobasal medium with $2 \%$ B-27 supplement, $10 \mathrm{ng} / \mathrm{ml}$ nerve growth factor, $1 \mathrm{mmol} / \mathrm{l} \mathrm{L-glutamine}$ and 1,000 U/ml penicillin/streptomycin/neomycin solution, which were all purchased from were purchased from Sigma Aldrich; Merck Millipore.

TUNEL assay. Dulbecco's Modified Eagle's medium/F12, FBS and neurobasal cell medium were purchased from Gibco; Thermo Fisher Scientific, Inc. The Apoptosis Detection System kit (Roche Diagnostics GmbH, Mannheim, Germany) was used for the TUNEL assay, according to the manufacturer's protocol. DRG neurons were seeded into 96-well plates at a density of $1 \times 10^{5}$ cells/well. After fixing with $4 \%$ paraformaldehyde for $1 \mathrm{~h}$, DRG neurons in each groups were washed with PBS and treated with $1 \%$ Triton X-100 in PBS for $30 \mathrm{~min}$ on ice. And then incubated for $60 \mathrm{~min}$ at $37^{\circ} \mathrm{C}$ with $50 \mu \mathrm{l}$ of TUNEL reaction mixture. Cells were subsequently incubated with DAPI (dilution, 1:800; Santa Cruz Biotechnology, Inc., Dallas, TX, USA) for $2 \mathrm{~h}$ at room temperature. After washing with PBS, the cells were analyzed by confocal microscopy (magnification, x40; Zeiss 510; Zeiss AG, Oberkochen, Germany). DRG neurons untreated with myristicin and/or hypoxia were used as a control group. To avoid counting the same cell in more than one region, the authors counted every fifth section (50 $\mu \mathrm{m}$ apart). For each plate, six fields of view were examined.

Western blotting. DRG neurons were washed twice with cold PBS and lysed in radioimmunoprecipitation assay buffer consisting of $50 \mathrm{mM}$ TRIS, $150 \mathrm{mM} \mathrm{NaCl}, 2 \%$ sodium dodecyl sulfate (SDS) and a protease inhibitor mixture (Roche Diagnostics $\mathrm{GmbH}$ ). Equal amounts of protein $(2.0 \mathrm{mg} / \mathrm{ml}, 10 \mu \mathrm{l}$ in each lane) were separated by $10 \%$ SDS-PAGE and electrophoretically transferred to polyvinylidene difluoride membranes. Membranes were blocked with $5 \%$ nonfat milk and incubated with primary antibodies at $4^{\circ} \mathrm{C}$ overnight. The following primary antibodies were used: B cell lymphoma 2 (cat. no. SAB4300339; dilution, 1:500; anti-rabbit; Sigma-Aldrich; Merck Millipore), Bcl-2 associated X protein (cat. no. SAB4502549; dilution, 1:800; anti-rabbit; Sigma-Aldrich; Merck Millipore), cleaved caspase-3 (cat. no. sc-22171; dilution, 1:500; anti-rabbit; Santa Cruz Biotechnology, Inc.), CHOP (cat. no. 2895P; dilution, 1:500; anti-rabbit; Cell Signaling Technology, Inc.), GRP78 (cat. no. G9043; dilution, 1:800; anti-rabbit; Sigma-Aldrich; Merck Millipore), cleaved caspase-12 (cat. no. sc-70227; dilution, 1:500; anti-rabbit; Santa Cruz Biotechnology, Inc.) and $\beta$-actin (cat. no. sc-47778; dilution, 1:1,000; anti-rabbit; Santa Cruz Biotechnology, Inc.). Membranes were subsequently 
incubated with a horseradish peroxidase-conjugated mouse anti-rabbit secondary antibody (cat. no. sc-2357; dilution, 1:5,000, Santa Cruz Biotechnology, Inc.) for $2 \mathrm{~h}$ at room temperature. Following each incubation, membranes were extensively washed in TBS containing Tween-20 and the immunoreactive bands were detected using an enhanced chemiluminescence kit (cat. no. orb90503; Biorbyt, Ltd.). The quantification of Western blotting was conducted using a computerized image-analysis system (Image Pro Plus; version, 6.0; Media Cybernetics, Inc., Rockville, MD, USA) in duplicate.

Detection of lactate dehydrogenase ( $L D H)$, superoxide dismutase (SOD), glutathione peroxidase (GSH-PX) and malondialdehyde (MDA). The DRG neurons were randomly divided into four groups: Control group, control plus $\mathrm{Myr}$ group, hypoxia group, and hypoxia plus Myr group. DRG neurons were seeded at $5 \times 10^{4}$ per well in 6 -well plates, cells were harvested and washed twice with cold PBS, before being lysed in radioimmunoprecipitation assay buffer consisting of $50 \mathrm{mM}$ TRIS, $150 \mathrm{mM} \mathrm{NaCl}, 2 \%$ SDS and a protease inhibitor mixture (Roche Diagnostics GmbH). DRG neurons were quantified using the bicinchoninic acid kit for protein determination (cat. no. BCA1-1KT; Sigma-Aldrich; Merck Millipore). SOD (cat. no. 20080829), GSH-PX (cat. no. 20080801) and MDA (cat. no. 20080801) levels in the supernatant were measured using commercially available kits purchased from Nanjing Jiancheng Bioengineering Institute (Nanjing, China). All assays were conducted according to the manufacturer's protocol.

$L D H$ release assay. $\mathrm{LDH}$ activity was evaluated using a colorimetric LDH assay. DRG neurons were seeded onto a 96-well plate under a $1 \% \mathrm{O}_{2}$ environment for $24 \mathrm{~h}$. Briefly, $100 \mu \mathrm{l}$ supernatant was transferred from each well to a new 96-well plate and $100 \mu 1$ fresh reaction mixture was added to each well. After $30 \mathrm{~min}$ of incubation at room temperature in the dark, the optical density values were detected at a wavelength of $490 \mathrm{~nm}$ using a microplate reader (Thermo Labsystems, Santa Rosa, CA, USA). The quantity of LDH was calculated as a percentage compared with the total amount of LDH present in cells treated with $1 \%$ Triton-X 100 (Beijing Solarbio Science \& Technology Co., Ltd., Beijing, China). LDH levels were measured using a commercially available kit (cat. no. 20071129) purchased from Nanjing Jiancheng Bioengineering Institute (Nanjing, China).

Reverse transcription-quantitative polymerase chain reaction $(R T-q P C R)$. Total RNA was extracted using TRIzol ${ }^{\mathbb{B}}$ solution (Invitrogen; Thermo Fisher Scientific, Inc.) according to the manufacturer's protocol. The quantity and quality of isolated RNA were evaluated using absorbance at wavelengths of 260 and $280 \mathrm{~nm}$. Following this, RT was performed in a $20 \mu 1$ reaction mixture using the RevertAid First Strand cDNA Synthesis kit (Thermo Fisher Scientific, Inc.). Primer sequences were as follows: Forward, 5'-GGA CCTGACCTGCCGTCTAG-3' and reverse, 5'-GTAGCCCAG GATGCCCTTGA-3' for $\beta$-actin; forward, 5'-GGUAUGAGG ACCUGCAAGA-3' and reverse, 5'-CACCAAGCAUGAACA AUUG-3' for CHOP; forward 5'-CUACCCAAACAUCGG

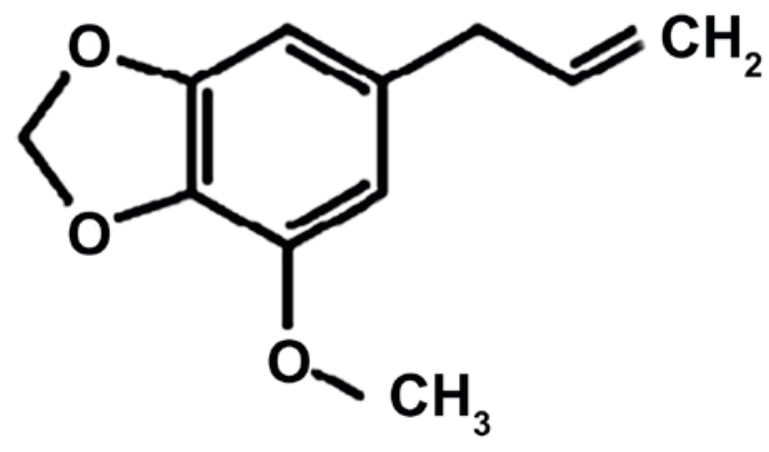

Figure 1. Chemical structure of myristicin.

GAAA-3' and reverse, 5'-CUCCAGAGAUGCUGAGCGA-3' for GRP78. qPCR with SYBR $^{\circledR}$ Green I (Shanghai Hi-Tech Enterprise Bio Co., Shanghai, China) (http://china.53trade. com/web0/disp_company_info.asp?userid=166543) was performed using a 7500 Real-Time PCR system (Applied Biosystems; Thermo Fisher Scientific, Inc.) with thermocycling conditions as follows: An initial denaturation step at $95^{\circ} \mathrm{C}$ for $5 \mathrm{~min}$, followed by 40 cycles of denaturation at $95^{\circ} \mathrm{C}$ for $15 \mathrm{sec}$, annealing at $60^{\circ} \mathrm{C}$ for $60 \mathrm{sec}$ and extension at $72^{\circ} \mathrm{C}$ for $30 \mathrm{sec}$. All samples were processed in triplicate. The mRNA expression levels of each gene were calculated using the relative quantitative method (23).

Immunofluorescence. DRG neurons were fixed with $4 \%$ PFA for $1 \mathrm{~h}$, washed with PBS containing $0.1 \%$ Triton X-100 (PBST), and blocked for $30 \mathrm{~min}$ in PBST supplemented with $10 \%$ FBS. DRG neurons were subsequently incubated with cleaved antibodies against caspase-12 (cat. no. sc-70227; dilution, 1:800; anti-rabbit; Santa Cruz Biotechnology, Inc.) in the same solution overnight at $4^{\circ} \mathrm{C}$, washed and incubated with a mouse anti-rabbit IgG-HRP conjugated secondary antibody (cat. no. sc-2357; dilution, 1:5,000, Santa Cruz Biotechnology, Inc.) for $2 \mathrm{~h}$ at room temperature. Nuclei were stained with DAPI (1:800; Santa Cruz Biotechnology, Inc.). The cells were examined under a Leica fluorescence microscope (Leica Microsystems GmbH, Wetzlar, Germany).

Statistical analysis. All data are presented as the mean \pm standard deviation of at least three independent experiments. All statistical analyses were performed using SPSS software version 11.0 (SPSS, Inc., Chicago, IL, USA). Statistical comparisons between the different treatments were performed using one-way analysis of variance with Tukey's multiple comparison post-hoc test. $\mathrm{P}<0.05$ was considered to indicate a statistically significant difference.

\section{Results}

Myr enhances the cell viability of hypoxia-induced DRG neurons. DRG neurons were exposed to 10-200 $\mu \mathrm{M}$ Myr, the chemical structure of which is presented in Fig. 1, for $24 \mathrm{~h}$. Concentrations of $10-50 \mu \mathrm{M}$ did not affect cell viability; however, viability was reduced with concentrations $>50 \mu \mathrm{M}$ (Fig. 2A). Therefore, 10-50 $\mu \mathrm{M}$ Myr was selected to treat the $1 \% \mathrm{O}_{2}$ (hypoxia)-exposed DRG neurons for $24 \mathrm{~h}$. Myr inhibited the hypoxia-induced decreased cell viability of DRG 
A

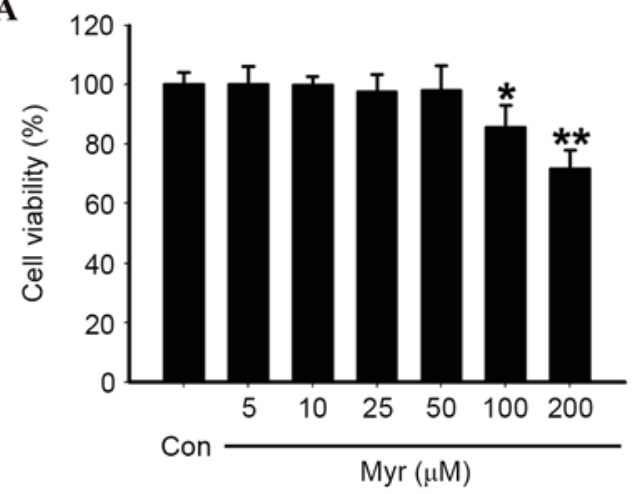

B

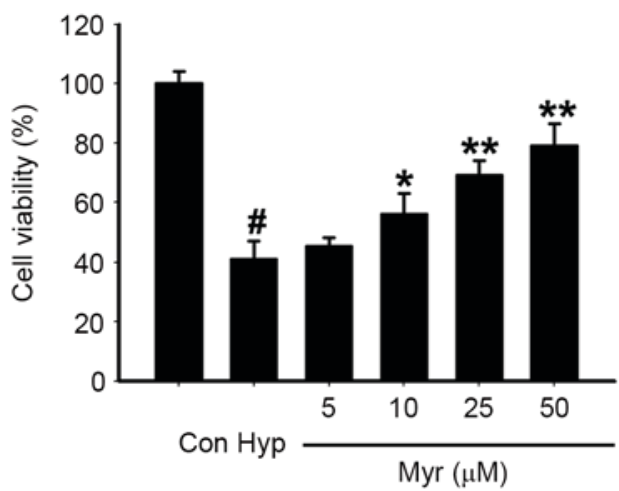

Figure 2. Effect of Myr on cell viability of hypoxia-induced DRG neurons. (A) The rat DRG neurons were exposed to 10 to $200 \mu \mathrm{M}$ Myr for $24 \mathrm{~h}$. Viability was significantly decreased at concentrations $>50 \mu \mathrm{M}$. ${ }^{*} \mathrm{P}<0.05$ and ${ }^{* *} \mathrm{P}<0.001$ vs. control group. (B) DRG neurons exposed to $1 \% \mathrm{O}_{2}$ (hypoxia) for $24 \mathrm{~h}$ had significantly decreased cell viability compared with control group; this was inhibited by Myr in a dose-dependent manner. Data are expressed as the mean \pm standard deviation. ${ }^{\#} \mathrm{P}<0.001$ vs. control; ${ }^{*} \mathrm{P}<0.05$ and ${ }^{* *} \mathrm{P}<0.001$ vs. hypoxia-induced group. Myr, myristicin; Con, control, DRG, dorsal root ganglion; Hyp, hypoxia.

neurons in a dose-dependent manner (Fig. 2B). The results confirmed that the optimal concentration of Myr was $50 \mu \mathrm{M}$.

Myr reverses the apoptosis of hypoxia-induced DRG neurons. TUNEL staining was used to assess apoptotic cells. As presented in Fig. 3A, increased TUNEL staining was observed in the hypoxia-induced DRG neurons compared with the normal group. The number of TUNEL-positive cells was reduced in DRG neurons following Myr treatment; however, Myr treatment had no effect on the normal group. Quantification of TUNEL staining revealed that Myr significantly reduced the percentage of TUNEL-positive DRG neurons following hypoxia (Fig. 3B).

To determine the possible involvement of the mitochondrial pathway of apoptosis in hypoxia-induced DRG neurons, protein expression levels of the pro-apoptotic Bax, the apoptosis protease cleaved caspase- 3 and the anti-apoptotic Bcl-2 were detected by western blot analysis (Fig 4A). Increased protein expression levels of Bax and cleaved caspase-3, and reduced protein expression levels of $\mathrm{Bcl}-2$, were detected in the hypoxia-induced group compared with the normal group; these effects were significantly abrogated by Myr treatment (Fig. 4B-D). Therefore, Myr may reverse hypoxia-induced apoptosis in DRG neurons by affecting the protein expression levels of apoptosis-associated molecules.

Myr decreases MDA content and LDH release, and upregulates SOD and GSH-PX activities, in hypoxia-induced DRG neurons. To investigate the effect of hypoxic injury on DRG neurons, the present study analyzed MDA content, LDH release and SOD and GSH-PX activities in the four groups. The MDA content (Fig. 5A) and LDH release (Fig. 5B) in hypoxia-induced DRG neurons was greater compared with the normal group, whereas SOD (Fig. 5C) and GSH-PX (Fig. 5D) activities were reduced. Following treatment with Myr, these hypoxia-induced effects were significantly attenuated. Therefore, Myr exhibited a protective effect against hypoxic injury in DRG neurons.

Myr reduces the expression levels of CHOP, GRP78 and cleaved caspase-12 in hypoxia-induced DRG neurons. The levels of CHOP and GRP78 were analyzed by western blot analysis (Fig. 6A) to determine whether ERS was involved in apoptosis of hypoxia-induced DRG neurons. CHOP (Fig. 6B) and GRP78 (Fig. 6C) protein expression levels were significantly increased in DRG neurons treated with hypoxia, and this increase was significantly inhibited by Myr. Similarly, CHOP (Fig. 6D) and GRP78 (Fig. 6E) mRNA expression levels in the hypoxia group were significantly increased compared with the normal group, as assessed by RT-qPCR; however, this was significantly downregulated by Myr. The protein expression levels of cleaved caspase-12 were analyzed as it is a specific marker of ERS-induced apoptosis (Fig. 7A). Western blot analysis indicated that hypoxia significantly increased its protein expression levels; however, this was significantly inhibited by treatment with Myr (Fig. 7B). Immunofluorescence verified this (Fig. 7C). These results suggested that Myr may inhibit hypoxia-ERS-induced apoptosis in DRG neurons.

\section{Discussion}

The biochemical cascade initiated by conditions of hypoxia is complex. Depletion of cellular energy reserves is a major problem (24) and the mitochondrial response to hypoxia is critical in determining neuronal cell fate (25). Severe tissue oxygen depletion may result in total mitochondrial failure and necrotic cell death, whereas less severe oxygen deprivation may trigger activation of the apoptotic pathway (26). Kerr et al (27) first described apoptosis as a programmed cell death defined by the nuclear and cell morphological alterations due to activation of signaling cascades. The results of the present study demonstrated the involvement of ERS in the apoptosis of hypoxia-induced DRG neurons.

Activation of the UPR protects cells under ERS via upregulation of GRP78 (28). Physiological processes that demand a high rate of protein synthesis and secretion must sustain activation of the adaptive programs of the UPR without triggering cell death pathways (29). However, prolonged activation of the UPR by excessive ERS may convert its role to a cytotoxic one, by activation of multiple apoptotic pathways in mammalian cells (30). The underlying mechanisms initiating apoptosis under conditions of irreversible ER damage are 
A

TUNEL
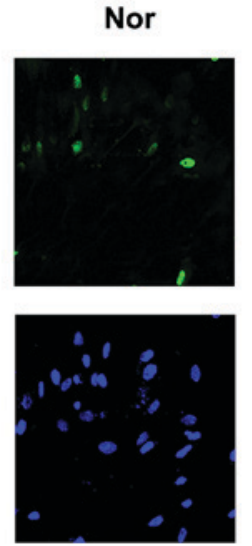

DAPI

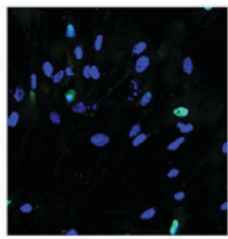

\section{Nor+Myr}
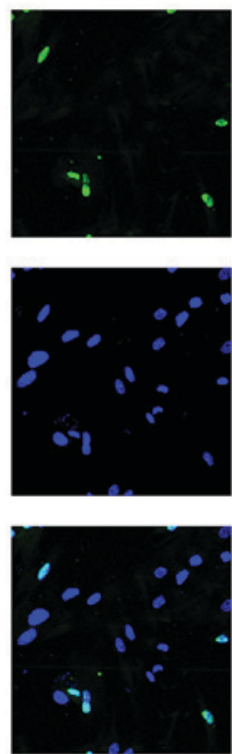

Hypoxia
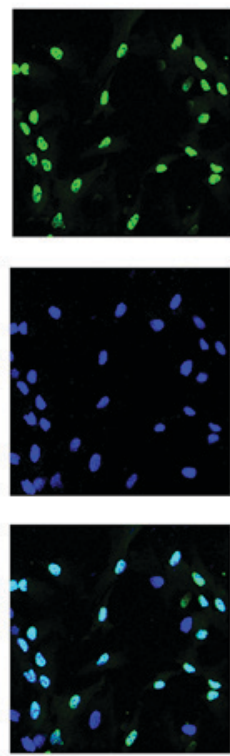

Hypoxia+Myr
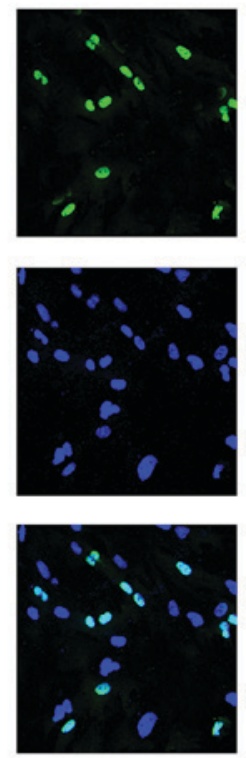
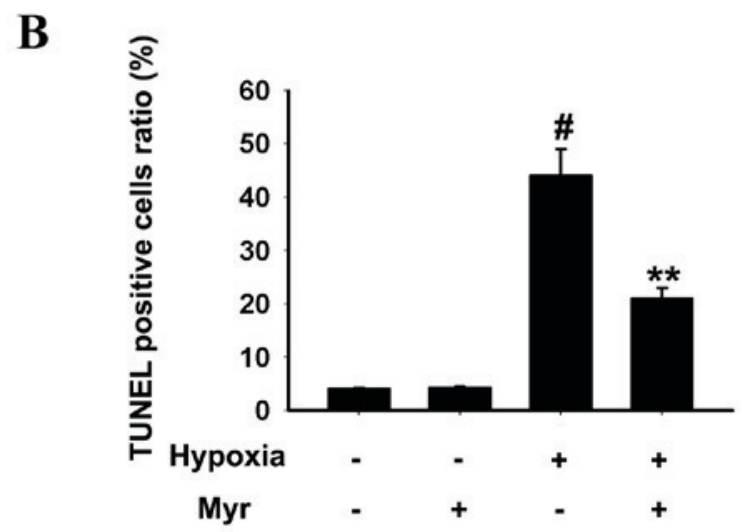

Figure 3. Myr reduces the number of TUNEL-positive DRG neurons following hypoxia. (A) TUNEL staining was performed in DRG neurons. The merged images, resulting from the overlap of TUNEL-positive (green) and DAPI-labeled (blue) areas, revealed increased TUNEL staining in the hypoxia group compared with the normal group after $24 \mathrm{~h}$; this increase was inhibited by $50 \mu \mathrm{M}$ Myr. (magnification, $\mathrm{x} 40$ ). (B) Quantification of TUNEL staining revealed that these differences were significant. Data were expressed as the mean \pm standard deviation. ${ }^{\prime \prime} \mathrm{P}<0.05$ vs. normal group; ${ }^{* *} \mathrm{P}<0.01$ vs. hypoxia-induced group. Myr, myristicin; DRG, dorsal root ganglion; TUNEL, deoxynucleotidyl transferase-mediated dUTP nick end-labeling; Nor, normal.

now partially understood and may involve a series of complementary pathways (29). The ERS transducer proteins ATF-6, IRE-1 $\alpha$ and PERK constitute the core stress regulators of the UPR, and transduce signals from the ER to the cytoplasm and nucleus following ERS (29). A previous study reported that the induction of ERS was closely associated with apoptosis (30). Chronic ER stress leads to Bax-dependent apoptosis through the transcriptional upregulation of Bcl-2 homology 3-only proteins, including $\mathrm{Bcl}-2$-interacting mediator of cell death (BIM) and p53 upregulated modulator of apoptosis, which are upstream Bcl-2 family members (31). The transcription of one of the key UPR pro-apoptotic genes, CHOP, is positively controlled by the PERK-ATF4 axis (31). CHOP promotes the transcription of BIM and the downregulation of Bcl-2 expression, contributing to the induction of apoptosis (31). The present study indicated that CHOP and GRP78 were expressed at high levels in hypoxia-induced DRG neurons, which increased the protein expression levels of Bax and decreased that of Bcl-2. Caspases are a superfamily of cysteine aspartyl-specific proteases that regulate numerous aspects of cell survival and death (32). Caspase-3 is a validated marker in detecting early neuronal apoptosis (32). Its activation is mediated via extrinsic (death ligand) and intrinsic (mitochondrial) pathways $(11,32$ ). The zymogen caspase- 3 has almost no activity until it is cleaved by an initiator caspase following apoptotic signaling events (11). The present study revealed that cleaved caspase-3 was expressed at high levels in hypoxia-induced DRG neurons. Caspase-12, which is localized on the ER membrane, has been demonstrated to be specifically activated by ER stress $(11,13)$. In the current study, the level of caspase-12 was significantly increased in hypoxia-induced DRG neurons, suggesting the involvement of caspase-12 in ERS-triggered cell apoptosis.

$\mathrm{LDH}$ is an enzyme that is present in the cell cytoplasm and is important in energy metabolism. An increase in LDH activity suggests cellular damage (33). Enhanced lipid peroxidation is a hallmark of free radical-induced tissue damage. The $\beta$-oxidation of lipid peroxides in cells leads to double bond rearrangement of unsaturated fatty acids of the 
A

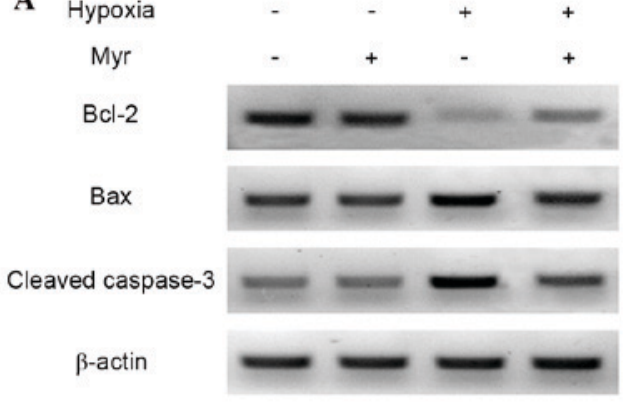

C

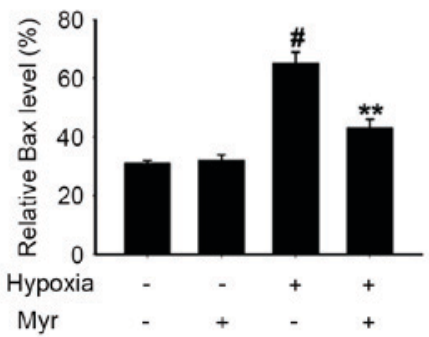

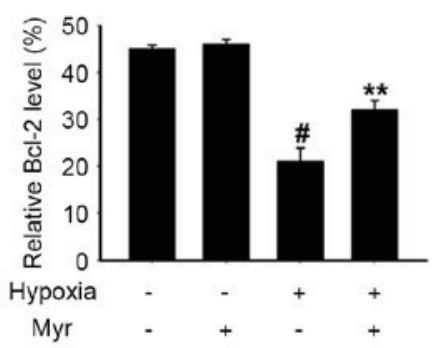

D

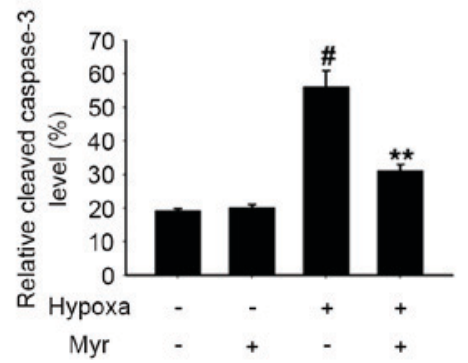

Figure 4. Myr influences the protein expression levels of Bcl-2, Bax and cleaved caspase-3 in hypoxia-induced DRG neurons. (A) Western blot analysis of DRG neurons following hypoxia and/or Myr treatment. Quantification of western blotting revealed that hypoxia treatment (B) decreased Bcl-2 protein expression levels compared with untreated cells, and increased protein expression levels of (C) Bax and (D) cleaved caspase-3. Myr treatment significantly inhibited these hypoxia-induced effects. $\beta$-actin served as a loading control. Data are expressed as the mean \pm standard deviation $(\mathrm{n}=3)$. ${ }^{*} \mathrm{P}<0.001 \mathrm{vs}$. untreated group; ${ }^{* *} \mathrm{P}<0.001$ vs. hypoxia-induced group. Myr, myristicin; DRG, dorsal root ganglion; Bcl-2, B cell lymphoma 2; Bax, Bcl-2 associated X protein.

A

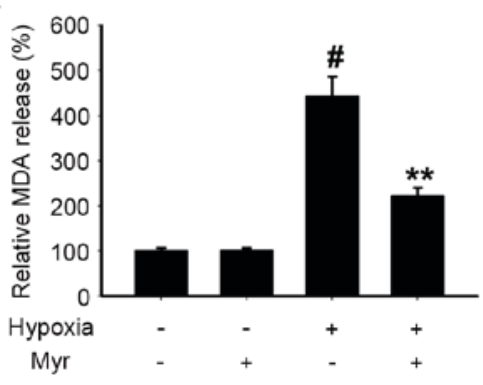

C

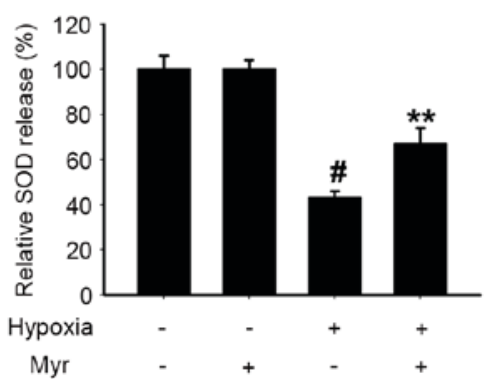

B

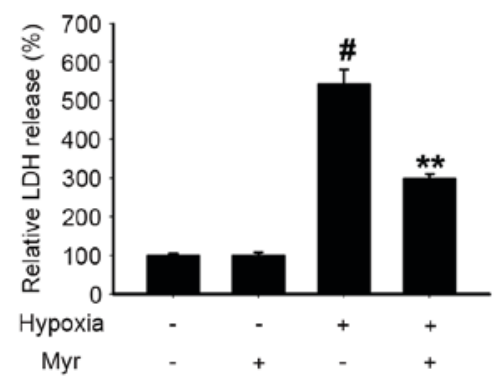

D

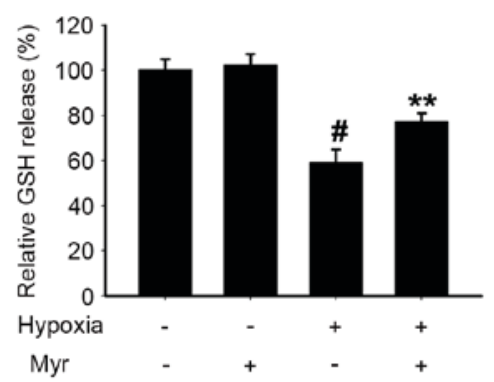

Figure 5. MDA, LDH, SOD and GSH-PX activity. Hypoxia treatment significantly increased (A) MDA content and (B) the release of LDH in DRG neurons compared with the untreated group; these effects were inhibited by Myr. Hypoxia-exposed DRG neurons had downregulated (C) SOD and (D) GSH-PX activities compared with untreated cells; again, these effects were inhibited by Myr. Data are expressed as the mean \pm standard deviation. ${ }^{~} \mathrm{P}<0.001$ vs. untreated group; " $\mathrm{P}<0.001$ vs. hypoxia-induced group. Myr, myristicin; DRG, dorsal root ganglion; MDA, malondialdehyde; LDH, lactate dehydrogenase; SOD, superoxide dismutase; GSH-PX, glutathione peroxidase.

membrane, which results in destruction of the lipid membrane culminating in tissue damage (33). This activity is enhanced due to stress exerted by free radicals (34). MDA is involved in the downregulation of lipid peroxidation. SOD reduces 
A

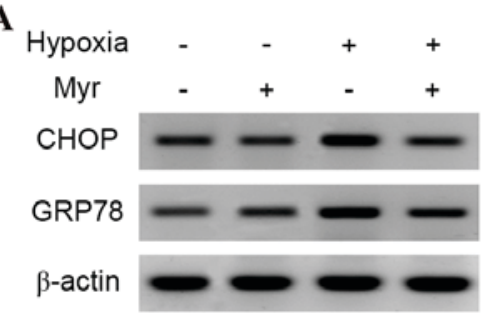

D
B

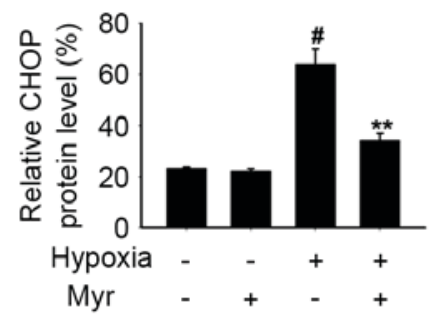

C

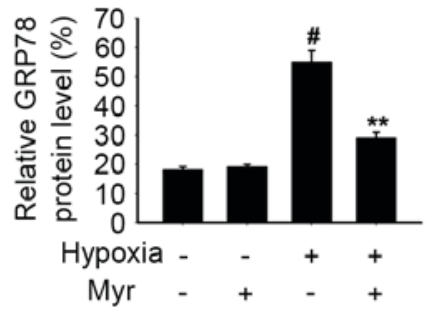

$\mathbf{E}$
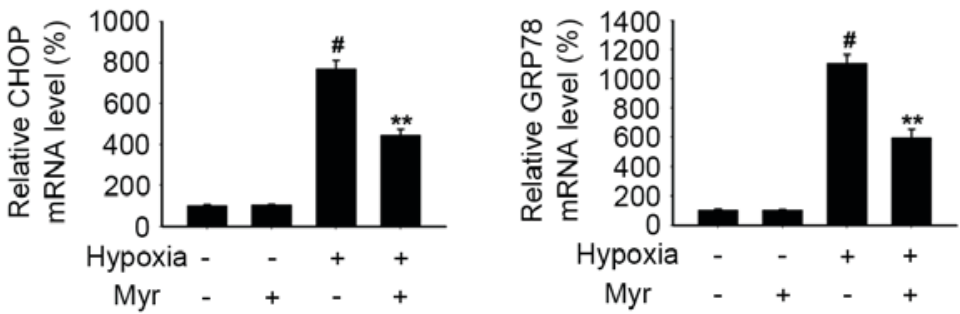

Figure 6. Myr decreases the expression of CHOP and GRP78 in DRG neurons exposed to hypoxia. (A) The levels of CHOP and GRP78 were analyzed using a western blot analysis. Protein expression levels of (B) CHOP and (C) GRP7 were significantly upregulated in DRG neurons exposed to hypoxia; however, addition of Myr significantly decreased their protein expression levels. $\beta$-actin served as a loading control. (D) CHOP and (E) GRP78 mRNA expression levels were detected by reverse transcription-quantitative polymerase chain reaction, and were enhanced in the hypoxia-induced group and inhibited by addition of Myr. Data are expressed as the mean \pm standard deviation. ${ }^{~} \mathrm{P}<0.001$ vs. untreated group; ${ }^{* *} \mathrm{P}<0.001$ vs. hypoxia-induced group. Myr, myristicin; $\mathrm{DRG}$, dorsal root ganglion; CHOP, CCAAT/enhancer-binding protein-homologous protein; GRP78, glucose-related protein 78.

A

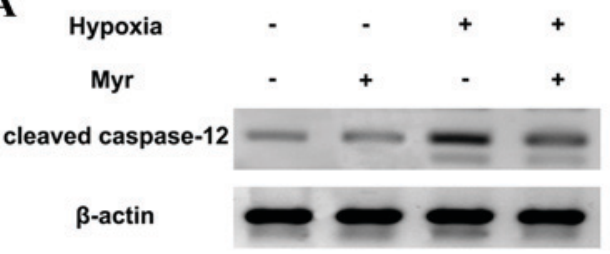

B

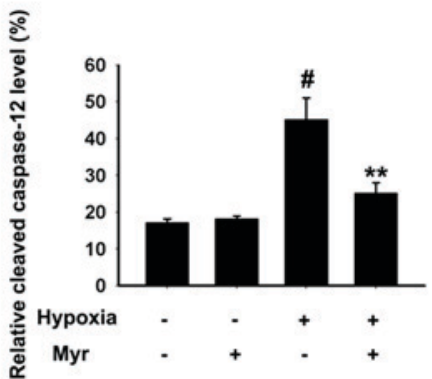

C

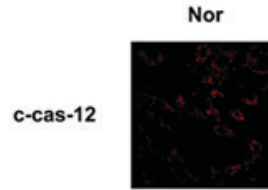

DAPI
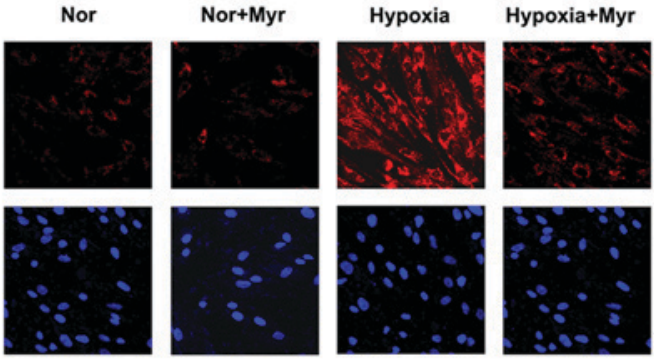

Merge
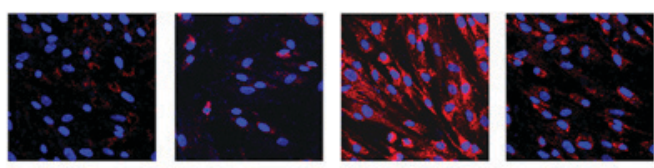

Figure 7. Myr reduces cleaved caspase-12 expression in hypoxia-induced DRG neurons. (A) Western blotting was performed to detect cleaved caspase-12 expression. (B) Quantification of western blotting revealed that protein expression levels of cleaved caspase-12 were significantly increased in hypoxia-induced DRG neurons compared with the untreated group; this effect was significantly inhibited by Myr. $\beta$-actin served as a loading control. (C) Immunofluorescence staining of cleaved caspase-12 verified the western blotting results. (magnification, $\mathrm{x} 40$ ). All data were expressed as the mean \pm standard deviation. ${ }^{\#} \mathrm{P}<0.001$ vs. normal group; ${ }^{* *} \mathrm{P}<0.001$ vs. hypoxia-induced group. Myr, myristicin; DRG, dorsal root ganglion.

$\mathrm{O}_{2}$ - to $\mathrm{H}_{2} \mathrm{O}$, thus scavenging noxious free radicals (35). GSH is an indispensable member of the antioxidant free radical scavenger family, which converts $\mathrm{H}_{2} \mathrm{O}_{2}$ to $\mathrm{H}_{2} \mathrm{O}$ (10). GSH shields cells and tissues against free radical generation during stressful conditions arising following the administration of acetic acid (10). GSH is important in electrophile detoxification, transport of amino acids and synthesis of DNA (10). The results of the present study demonstrated that hypoxia treatment significantly increased MDA content and the release of LDH, but downregulated SOD and GSH-PX activities in DRG neurons.

A variety of drugs targeting cell death pathways have been assessed in animal models of spinal cord injury. The amplitude of neuroprotection observed in these studies has been 
variable, and often the results are inconsistent between models and research groups. However, various compounds, including erythropoietin, $\mathrm{N}$-acetyl-cysteine, caspase-2 inhibitors, p53 inhibitors, melatonin and c-Jun N-terminal kinase inhibitors, have demonstrated promising neuroprotective properties. Myr, as the primary active constituent of nutmeg, mace and parsley leaf oil, has not been investigated in in vivo animal models of spinal cord injury or DRG neuron cell models. In conclusion, the present study, to the best of our knowledge, first demonstrated that Myr may protect against hypoxia-induced apoptosis in rat DRG neurons via inhibition of the ERS pathway. However, limitations of the present study include the fact that it remains unknown whether Myr is able to cross the blood-brain barrier. In addition, the safety of Myr needs to be carefully tested in long-term follow-up studies. Furthermore, the majority of drugs examined are non-specific and have multiple effects in addition to anti-apoptotic/antinecrotic effects. Further in vivo studies are required to test the effects of Myr in animal models and in humans.

\section{Acknowledgements}

The present study was supported by the National Natural Science Foundation of China (grant no. 81272048) and the Natural Science Foundation of Anhui Province (grant no. 1308085MH152).

\section{References}

1. Astorino TA, Harness ET and White AC: Efficacy of acute intermittent hypoxia on physical function and health status in humans with spinal cord injury: A brief review. Neural Plast 2015: 409625, 2015.

2. Navarrete-Opazo A, Vinit S, Dougherty BJ and Mitchell GS: Daily acute intermittent hypoxia elicits functional recovery of diaphragm and inspiratory intercostal muscle activity after acute cervical spinal injury. Exp Neurol 266: 1-10, 2015.

3. Kesherwani V, Atif F, Yousuf S and Agrawal SK: Resveratrol protects spinal cord dorsal column from hypoxic injury by activating Nrf-2. Neuroscience 241: 80-88, 2013.

4. Poltl D, Schildknecht S, Karreman C and Leist M: Uncoupling of ATP-depletion and cell death in human dopaminergic neurons. Neurotoxicology 33: 769-779, 2012.

5. Xavier JM, Morgado AL, Sola S and Rodrigues CM: Mitochondrial translocation of p53 modulates neuronal fate by preventing differentiation-induced mitochondrial stress. Antioxid Redox Signal 21: 1009-1024, 2014.

6. Brown JE, Zeiger SL, Hettinger JC, Brooks JD, Holt B Morrow JD, Musiek ES, Milne G and McLaughlin B: Essential role of the redox-sensitive kinase p66shc in determining energetic and oxidative status and cell fate in neuronal preconditioning. J Neurosci 30: 5242-5252, 2010.

7. Luo C, Li Q, Gao Y, Shen X, Ma L, Wu Q, Wang Z, Zhang M, Zhao Z, Chen X and Tao L: Poloxamer 188 attenuates cerebral hypoxia/ischemia injury in parallel with preventing mitochondrial membrane permeabilization and autophagic activation. J Mol Neurosci 56: 988-998, 2015.

8. Wu Y, Kazumura K, Maruyama W, Osawa T and Naoi M: Rasagiline and selegiline suppress calcium efflux from mitochondria by PK11195-induced opening of mitochondrial permeability transition pore: A novel anti-apoptotic function for neuroprotection. J Neural Transm (Vienna) 122: 1399-1407, 2015.

9. Zhu X, Zelmer A, Kapfhammer JP and Wellmann S: Cold-inducible RBM3 inhibits PERK phosphorylation through cooperation with NF90 to protect cells from endoplasmic reticulum stress. FASEB J 30: 624-634, 2016

10. Goswami P, Gupta S, Biswas J, Sharma S and Singh S Endoplasmic reticulum stress instigates the rotenone induced oxidative apoptotic neuronal death: A study in rat brain. Mol Neurobiol 53: 5384-5400, 2016.
11. Hetz C: The unfolded protein response: Controlling cell fate decisions under ER stress and beyond. Nat Rev Mol Cell Biol 13: 89-102, 2012

12. Bernales S, Soto MM and McCullagh E: Unfolded protein stress in the endoplasmic reticulum and mitochondria: A role in neurodegeneration. Front Aging Neurosci 4: 5, 2012.

13. Qiu B, Hu S, Liu L, Chen M, Wang L, Zeng X and Zhu S: CART attenuates endoplasmic reticulum stress response induced by cerebral ischemia and reperfusion through upregulating BDNF synthesis and secretion. Biochem Biophys Res Commun 436: 655-659, 2013

14. Farzaei MH, Abbasabadi Z, Ardekani MR, Rahimi R and Farzaei F: Parsley: A review of ethnopharmacology, phytochemistry and biological activities. J Tradit Chin Med 33: 815-826, 2013.

15. Martins C, Doran C, Silva IC, Miranda C, Rueff J and Rodrigues AS: Myristicin from nutmeg induces apoptosis via the mitochondrial pathway and down regulates genes of the DNA damage response pathways in human leukaemia K562 cells. Chem Biol Interac 218: 1-9, 2014.

16. Jabrane A, Ben Jannet H, Mastouri M, Mighri Z and Casanova J: Chemical composition and in vitro evaluation of antioxidant and antibacterial activities of the root oil of Ridolfia segetum (L.) Moris from Tunisia. Nat Prod Res 24: 491-499, 2010.

17. Morita T, Jinno K, Kawagishi H, Arimoto Y, Suganuma H, Inakuma T and Sugiyama K: Hepatoprotective effect of myristicin from nutmeg (Myristica fragrans) on lipopolysaccharide/d-galactosamine-induced liver injury. J Agric Food Chem 51: 1560-1565, 2003.

18. Lee JY and Park W: Anti-inflammatory effect of myristicin on RAW 264.7 macrophages stimulated with polyinosinic-polycytidylic acid. Molecules 16: 7132-7142, 2011.

19. Lee BK, Kim JH, Jung JW, Choi JW, Han ES, Lee SH, Ko KH and Ryu JH: Myristicin-induced neurotoxicity in human neuroblastoma SK-N-SH cells. Toxicol Lett 157: 49-56, 2005.

20. Chen F, Wu R, Zhu Z, Yin W, Xiong M, Sun J, Ni M, Cai G and Zhang $\mathrm{X}$ : Wogonin protects rat dorsal root ganglion neurons against tunicamycin-induced ER stress through the PERK-eIF2a-ATF4 signaling pathway. J Mol Neurosci 55: 995-1005, 2015.

21. Chen S, Xiong J, Zhan Y, Liu W and Wang X: Wogonin inhibits LPS-induced inflammatory responses in rat dorsal root ganglion neurons via inhibiting TLR4-MyD88-TAK1-mediated NF-aB and MAPK signaling pathway. Cell Mol Neurobiol 35: 523-531, 2015.

22. Xu S, Zhao X, Zhao Q, Zheng Q, Fang Z, Yang X, Wang H, Liu P and $\mathrm{Xu} \mathrm{H}$ : Wogonin prevents rat dorsal root ganglion neurons death via inhibiting tunicamycin-induced ER stress in vitro. Cell Mol Neurobiol 35: 389-398, 2015.

23. Livak KJ and Schmittgen TD: Analysis of relative gene expression data using real-time quantitative PCR and the 2(-Delta Delta C(T)) Method. Methods 25: 402-408, 2001.

24. Ponsuksili S, Jonas E, Murani E, Phatsara C, Srikanchai T, Walz C, Schwerin M, Schellander K and Wimmers K: Trait correlated expression combined with expression QTL analysis reveals biological pathways and candidate genes affecting water holding capacity of muscle. BMC Genomics 9: 367, 2008.

25. Nijboer CH, Bonestroo HJ, Zijlstra J, Kavelaars A and Heijnen CJ: Mitochondrial JNK phosphorylation as a novel therapeutic target to inhibit neuroinflammation and apoptosis after neonatal ischemic brain damage. Neurobiol Dis 54: 432-444, 2013.

26. Rau TF, Lu Q, Sharma S, Sun X, Leary G, Beckman ML, Hou Y, Wainwright MS, Kavanaugh M, Poulsen DJ and Black SM: Oxygen glucose deprivation in rat hippocampal slice cultures results in alterations in carnitine homeostasis and mitochondrial dysfunction. PLoS One 7: e40881, 2012.

27. Kerr JF, Wyllie AH and Currie AR: Apoptosis: A basic biological phenomenon with wide-ranging implications in tissue kinetics. Br J Cancer 26: 239-257, 1972.

28. Ranganathan AC, Ojha S, Kourtidis A, Conklin DS and Aguirre-Ghiso JA: Dual function of pancreatic endoplasmic reticulum kinase in tumor cell growth arrest and survival. Cancer Res 68: 3260-3268, 2008.

29. Glover-Cutter KM, Lin S and Blackwell TK: Integration of the unfolded protein and oxidative stress responses through SKN-1/Nrf. PLoS Genet 9: e1003701, 2013.

30. Mishra R and Karande AA: Endoplasmic reticulum stress-mediated activation of p38 MAPK, Caspase-2 and Caspase-8 leads to abrin-induced apoptosis. PLoS One 9: e92586, 2014.

31. Woehlbier U and Hetz C: Modulating stress responses by the UPRosome: A matter of life and death. Trends Biochem Sci 36: 329-337, 2011. 
32. Kanbak G, Kartkaya K, Ozcelik E, Guvenal AB, Kabay SC, Arslan G and Durmaz R: The neuroprotective effect of acute moderate alcohol consumption on caspase-3 mediated neuroapoptosis in traumatic brain injury: The role of lysosomal cathepsin L and nitric oxide. Gene 512: 492-495, 2013.

33. Venkataranganna MV, Rafiq M, Gopumadhavan S, Peer G, Babu UV and Mitra SK: NCB-02 (standardized Curcumin preparation) protects dinitrochlorobenzene- induced colitis through down-regulation of NFkappa-B and iNOS. World J Gastroenterol 13: 1103-1107, 2007.
34. Kandhare AD, Raygude KS, Ghosh P, Ghule AE, Gosavi TP, Badole SL and Bodhankar SL: Effect of hydroalcoholic extract of Hibiscus rosa sinensis Linn. leaves in experimental colitis in rats. Asian Pac J Trop Biomed 2: 337-344, 2012.

35. Kruidenier L, Kuiper I, Van Duijn W, Mieremet-Ooms MA, van Hogezand RA, Lamers CB and Verspaget HW: Imbalanced secondary mucosal antioxidant response in inflammatory bowel disease. J Pathol 201: 17-27, 2003. 